\title{
Primer registro de la especie exótica Pieris brassicae (Linnaeus, 1758) (Lepidoptera: Pieridae) en la Región de Atacama, Chile
}

\author{
First record of exotic species Pieris brassicae (Linnaeus, 1758) (Lepidoptera: \\ Pieridae) in the region of Atacama, Chile \\ Álvaro Zúñiga-Reinoso ${ }^{1 *}$, Daniela Mardones ${ }^{2}$
}

\section{RESUMEN}

Se da a conocer un nuevo registro de la mariposa de la col Pieris brassicae (L.) en Chile. Se extiende el rango de distribución septentrional y es el primer registro para la Región de Atacama.

Palabras clave: Brassicaceae, invasiones, mariposa de la col, nuevo registro, Huasco.

\begin{abstract}
Disclosed is a new record of the large white butterfly (Pieris brassicae) from Chile. The northern distribution range is extended and is the first record for the region of Atacama.
\end{abstract}

Key words: Brassicaceae, Invasions, large white butterfly, new record, Huasco.

Pieris brassicae (Linneo, 1758) es una mariposa nativa de Europa cuyo nombre común es "mariposa de la col". Esta especie ingresa a Chile de forma accidental en los años 70 por Valparaíso (Gardiner 1974), probablemente en cargamentos de hortalizas provenientes de ese continente (Peña \& Ugarte, 1997). Esta mariposa puede ser considerada una especie invasora, ya que puede causar daños en los cultivos de crucíferas y umbelíferas (Feltwell, 1978) y daños en las poblaciones de mariposas nativas de los países donde se ha introducido (Herrera, 1982). En Chile esta especie se ha documentado desde Valdivia hasta Coquimbo (Peña \& Ugarte, 1997) y trabajos más recientes siguen documentando la misma distribución (Benyamini et al., 2014). Sin embargo, durante el 2002 se recolecta material de esta especie al norte de Coquimbo, por lo que este manuscrito tiene como objetivo dar a conocer este nuevo registro.

Material examinado: Chile. Provincia de Huasco. Huasco Bajo (28 $27^{\prime}$ 'S; 71¹0’O). 15.8.2002. leg. A. Zúñiga. (1ㅇํ수). (ex. yuyo y rábano silvestre).

La literatura menciona que el límite norte de la distribución de P. brassicae en Chile correspondería a la zona de Coquimbo (Peña \& Ugarte, 1997; Beyamini et al., 2014). Por lo tanto, este reporte corresponde a un nuevo registro que extiende su rango septentrional, siendo además el primer registro de esta especie para la región de Atacama. Ambos ejemplares fueron recolectados en una plantación de olivos, asociados a las malezas Brassica rapa L. y Raphanus sativus L. (Brassicaceae), donde el ejemplar hembra fue captada depositando huevos en las hojas R. sativus (Figura 1).

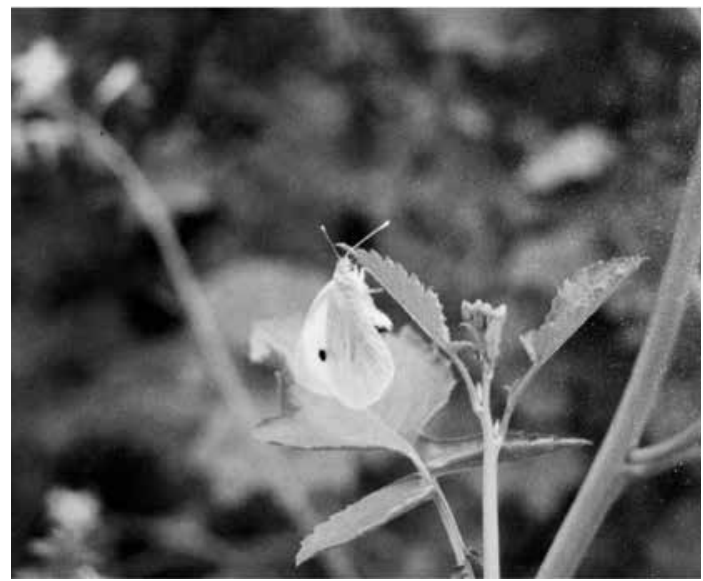

Figura 1. Hembra de Pieris brassicae depositando huevos bajo la faz inferior de la hoja de Raphanus sativus.

1 Programa de Doctorado en Ecología y Biología Evolutiva, Departamento de Ciencias Ecológicas, Facultad de Ciencias, Universidad de Chile.

2 Departamento de Botánica, Facultad de Ciencias Naturales y Oceanográficas, Universidad de Concepción.

* Autor para correspondencia: alzure@ gmail.com 
Cabe destacar que la familia Brassicaceae es donde se desarrollan las larvas de P. brassicae, lo que le da su nombre común de mariposa de la col (Peña \& Ugarte, 1996), además $R$. sativus es considerada una especie exótica establecida en la región (Fuentes et al., 2012), por lo que existiría el hábitat propicio para el establecimiento de esta especie en la región. Por lo tanto, el establecimiento de plantas exóticas naturalizadas podrían ayudar al establecimiento de insectos con ciclo de vidas relacionados a estas plantas.
Finalmente, reportes como este son información básica y un buen mecanismo de seguimiento para estudiar procesos de invasión y realizar planes de manejo y control de especies invasoras en Chile.

\section{Agradecimientos}

AZR agradece a beca CONICYT $21110367 \mathrm{y}$ DM agradece a beca CONICYT 22131484.

\section{Literatura Citada}

Benyamini B.; A. Ugarte; A.M. Shapiro; O.H. Hendrik; T. Pyrcz; Z. Bálint

2014. An updated list of the butterflies of Chile (Lepidoptera, Papilionoidea and Hesperioidea) including distribution, flight period and conservation status part I, comprising the families: Papilionidae, Pieridae, Nymphalidae (in part) and Hesperiidae describing a new species of Hypsochila (Pieridae) and a new subspecies of Yramea modesta (Nymphalidae). Boletín del Museo Nacional de Historia Natural, 63: 9-31.

Feltwell, J.

1978. The depredation of the Large White Butterfly (Pieris brassicae) (Pieridae). Journal or Reaserch on the Lepidoptera, 17: 218-225.
Fuentes, N.; A. Pauchard; P. Sánchez; J. Esquivel; A. Marticorena 2012. A new comprehensive database of alien plant species in Chile based on herbarium records. Biological Invasions, 15: 847-858.

Gardiner. B.O.

1974. Pieris brassicae L. established in Chile; another palearctic pest crosses the atlantic (Pieridae). Journal of the Lepidopterists' Society, 28: 269-277.

Herrera, J.

1982 . La vida silvestre: ¿Se extinguen las mariposas en Chile? Academia Superior de Ciencias Pedagógicas de Santiago. Rev. Academia, 3: 203-232.

Peña, L.; A. Ugarte 1997. Las Mariposas de Chile. Editorial Universitaria. Santiago-Chile. pp. 359. 